\title{
Poisson versus threshold models for genetic analysis of clinical mastitis in US Holsteins
}

\author{
A. I. Vazquez, ${ }^{* 1}$ K. A. Weigel, ${ }^{\star}$ D. Gianola, ${ }^{\star} † \ddagger$ D. M. Bates,§ M. A. Perez-Cabal,\# G. J. M. Rosa, ${ }^{*}$ \\ and Y. M. Chang\| \\ *Department of Dairy Science, \\ †Department of Animal Sciences, \\ fDepartment of Biostatistics and Medical Informatics, and \\ §Department of Statistics, University of Wisconsin, Madison 53706 \\ \#Departamento de Producción Animal, Universidad Politécnica de Madrid, 28040, Madrid, Spain \\ $\|$ Section of Epidemiology and Biostatistics, Leeds Institute of Molecular Medicine, Leeds, United Kingdom
}

\section{ABSTRACT}

Typically, clinical mastitis is coded as the presence or absence of disease in a given lactation, and records are analyzed with either linear models or binary threshold models. Because the presence of mastitis may include cows with multiple episodes, there is a loss of information when counts are treated as binary responses. Poisson models are appropriated for random variables measured as the number of events, and although these models are used extensively in studying the epidemiology of mastitis, they have rarely been used for studying the genetic aspects of mastitis. Ordinal threshold models are pertinent for ordered categorical responses; although one can hypothesize that the number of clinical mastitis episodes per animal reflects a continuous underlying increase in mastitis susceptibility, these models have rarely been used in genetic analysis of mastitis. The objective of this study was to compare probit, Poisson, and ordinal threshold models for the genetic evaluation of US Holstein sires for clinical mastitis. Mastitis was measured as a binary trait or as the number of mastitis cases. Data from 44,908 first-parity cows recorded in on-farm herd management software were gathered, edited, and processed for the present study. The cows were daughters of 1,861 sires, distributed over 94 herds. Predictive ability was assessed via a 5-fold cross-validation using 2 loss functions: mean squared error of prediction (MSEP) as the end point and a cost difference function. The heritability estimates were 0.061 for mastitis measured as a binary trait in the probit model and 0.085 and 0.132 for the number of mastitis cases in the ordinal threshold and Poisson models, respectively; because of scale differences, only the probit and ordinal threshold models are

Received January 28, 2009.

Accepted June 29, 2009.

${ }^{1}$ Corresponding author: aivazquez@wisc.edu directly comparable. Among healthy animals, MSEP was smallest for the probit model, and the cost function was smallest for the ordinal threshold model. Among diseased animals, MSEP and the cost function were smallest for the Poisson model, followed by the ordinal threshold model. In general, the models for count variables more accurately identified diseased animals and more accurately predicted mastitis costs. Healthy animals were more accurately identified by the probit model.

Key words: mastitis, Poisson model, ordinal threshold model, probit model

\section{INTRODUCTION}

In recent decades, genetic selection of dairy cattle in the United States has focused mainly on increasing milk yield. Average annual milk yield per cow in the United States almost doubled in $40 \mathrm{yr}$, from approximately $6,300 \mathrm{~kg}$ in 1960 to $11,700 \mathrm{~kg}$ in 2000 , with a large portion of the increase caused by genetic improvement (http://aipl.arsusda.gov). Antagonistic genetic correlations between milk production and disease traits, such as mastitis, have been reported frequently in the literature (e.g., Heringstad et al., 2000). In the United States, there is no national recording system at present that allows direct genetic evaluation of resistance to clinical mastitis, so data on SCS are used instead (Shook, 1982). However, many farmers collect disease data in their on-farm herd management software programs. Data codes used to represent specific diseases vary between farms and between software programs. Zwald (2004) and Zwald et al. (2004) listed the plausible codes that were used for mastitis and other diseases in a group of 418 US dairy herds. Although the criteria for diagnosing and categorizing specific infectious diseases and metabolic disorders vary widely between farms, these data can be useful for predicting the genetic merit of dairy sires for the susceptibility of their daughters to 
common health disorders, because semen from AI sires is typically distributed across many herds.

Statistical analysis of clinical mastitis with linear models is the most common method used for genetic evaluation, with mastitis coded as present or absent during a certain period. Threshold models (Gianola and Foulley, 1983) have also been applied, assuming a continuous underlying variable called liability, which represents the susceptibility of a cow to mastitis infection. The observed outcome is the presence or absence of the clinical form of the disease, and the probit (or threshold) model postulates that if liability exceeds a certain threshold, the cow will develop mastitis. Other models for longitudinal data that consider the time at which mastitis infection occurs have been applied by using survival analysis models (Saebo et al., 2002), longitudinal probit models (Rekaya et al., 1998), and multivariate probit models (Heringstad et al., 2004). Genetic analyses of clinical mastitis have rarely considered the number of cases in a lactation period by using a Poisson regression model (Vazquez et al., 2009) or an ordinal threshold model (Heringstad et al., 2006). Recently, the Poisson model was compared with a binomial model with a logit link in Norwegian Red cattle (Vazquez et al., 2009) and with a binomial model using the probit link in Spanish Holstein cows (Vazquez, 2007).

The objective of this study was to compare Poisson, ordinal threshold, and probit modeling strategies for genetic analysis of clinical mastitis (as either the number of cases or as the absence or presence of disease) by using farmer-recorded data in US Holsteins. Specifically, we evaluated and compared the predictive abilities and the difference in estimated and real mastitis costs of the models.

\section{MATERIALS AND METHODS}

\section{Data}

Data from the Advantage progeny test herds of Alta Genetics (Watertown, WI) were collected from 2000 through 2005 by using either Dairy Comp 305 (Valley Ag Software, Tulare, CA), PCDART (DRMS, Raleigh, NC), or DHI-Plus (DHI-Provo, Provo, UT) herd management software. Alta Genetics staff recovered these data in herd visits every other month. Data consisted of 54,137 first-lactation cows. The coding of disease information differed between farms, and the list of plausible codes developed by Zwald (2004) was used to identify the mastitis incidence records. An additional list of codes was developed to capture cases of mastitis recorded under the more general events "ill," "hospital," "disease," "treat," "died," or "better," based on corresponding remarks about the specific treatment that was applied in each case. The incidence rate was calculated for each herd, and edits were applied as follows: 1) herds with an incidence rate of less than $1 \%$ for 3 or more years were deleted; 2 ) herds with an incidence rate of less than $1 \%$ in 1 or $2 \mathrm{yr}$, plus a total incidence rate of less than $1 \%$ were also deleted; 3 ) multiple records of mastitis within a period of fewer than 6 consecutive days for an individual cow were assumed to describe the same case (Gasqui and Barnouin, 2003); and 4) each sire and herd was required to have a minimum of 5 observations. After editing, data consisted of 44,908 first-lactation cows, which were daughters of 1,861 sires, housed in 94 herds.

Overall, $84.4 \%$ of the cows were healthy, $11.1 \%$ had 1 case of mastitis, $2.6 \%$ had 2 cases, $1 \%$ had 3 cases, $0.4 \%$ had 4 cases, and $0.5 \%$ had 5 or more cases. Mastitis incidence in first-lactation cows in this population was low (15.6\%). Many sires (28\%) had daughters without any incidence of mastitis.

\section{Statistical Models}

Response variables were the number of clinical mastitis reports in the first lactation (NMAST) and a binary variable (BINMAST) denoting the presence or absence of a clinical mastitis infection during the first lactation.

The following linear predictor was common to all models:

$$
\eta_{i j k}=\alpha+C Y_{i}+S_{j}+H_{k}
$$

where $\eta_{i j k}$ is a function of the expected NMAST or BINMAST in the first lactation of a specific cow; $\alpha$ is an intercept; $C Y_{i}$ is the calving year $(i=1, \ldots, 6) ; S_{j}$ is the random transmitting ability of the $j$ th sire $(j=$ $1, \ldots, 1,861$ sires); and $H_{k}$ is the random effect of the $k$ th herd $(k=1, \ldots, 94)$. The random effects were assumed to follow a multivariate normal distribution:

$$
\left(\begin{array}{l}
\mathbf{s} \\
\mathbf{h}
\end{array}\right) \sim N\left[\mathbf{0},\left(\begin{array}{cc}
\mathbf{A} \sigma_{s}^{2} & \mathbf{0} \\
\mathbf{0} & \mathbf{I} \sigma_{h}^{2}
\end{array}\right),\right.
$$

where $\mathbf{s}$ and $\mathbf{h}$ are the vectors of sire and herd effects, respectively; $\sigma_{s}^{2}$ and $\sigma_{h}^{2}$ are the between-sire and between-herd variance components, respectively; $\mathbf{A}$ is the additive genetic relationship matrix between sires; and I is an identity matrix of 94 rows and columns. Sire and herd effects were assumed to be independent of residuals in the models. In the threshold models, a random residual effect was assumed to be normally and independently distributed, with mean 0 and variance 1 . The 
residual variance was set to 1 , because this parameter is not identifiable. Hence, all effects were measured in units of residual standard deviation.

Probit Model. The BINMAST was analyzed with a mixed effects probit model (Gianola and Foulley, 1983). This model describes variation at the level of an underlying random variable $z$ (liability). Above a certain unknown threshold, the outcome for mastitis was "sick" and below that threshold, the outcome was "healthy." In a binary response situation, the threshold was set to zero, such that

$$
\text { BINMAST }_{i j k l}=\left\{\begin{array}{ll}
1 & \text { if } z_{i j k l}>0 \\
0 & \text { otherwise }
\end{array} .\right.
$$

The model for the latent variable was

$$
z_{i j k l}=\eta_{i j k}+e_{i j k l}
$$

where $z_{i j k l}$ is the unobserved liability to mastitis of daughter $l$ of sire $j$, calving year $i$ in herd $k$, and $e_{i j k l}$ is the random residual component associated with the observation from daughter $l$.

It follows that the conditional distribution of BINMAST was

$$
\begin{aligned}
& \text { BINMAST } \\
& \text { ijkl } \mid \text { fixed and random effects } \\
& \sim \text { Bernoulli }\left[\boldsymbol{\Phi}\left(\eta_{i j k}\right)\right]
\end{aligned}
$$

where $\boldsymbol{\Phi}($.$) is the standard normal cumulative distribu-$ tion function. Given the random effects, all mastitis records were assumed to be independent, so the joint distribution of the observed data was the product of Bernoulli distributions.

Ordinal Threshold Model. The NMAST can be viewed as an ordered categorical response, so a mixed effects ordinal threshold model was fitted. The ordinal threshold model is appropriate for ordinal categorical data with more than 2 categories, as long as there are enough observations in each category. Again, the threshold model postulates a latent variable for each observation (Gianola, 1982) and the response observed takes the value $m:\{0,1,2,3,4,5$ or more $\}$ cases of mastitis in the first lactation if the latent variable falls between thresholds $T_{m-1}$ and $T_{m}$. Heringstad et al. (2006) used ordinal threshold models for sampling the liability of animals with censored categorical records in a Bayesian Markov chain Monte Carlo context.

The mixed effects ordinal-threshold model for NMAST was

$$
\begin{gathered}
\operatorname{Pr}\left(N M A S T_{i j k}=m \mid \text { fixed and random effects }\right) \\
=\boldsymbol{\Phi}\left[T_{m}-\left(\eta_{i j k}\right)\right]-\boldsymbol{\Phi}\left[T_{m-1}-\left(\eta_{i j k}\right)\right],
\end{gathered}
$$

where $m=0,1, \ldots, 5$ indexes the category of response (see above); $\boldsymbol{\Phi}($.$) is the standard normal cumulative$ distribution function of the standard normal, and $T_{m}$ and $T_{m-1}$ are fixed thresholds satisfying the order constraint $-\infty<T_{0}<T_{1}<\ldots<T_{m}<\infty$. The first threshold $\left(T_{0}\right)$ is set to zero, because constraint is necessary to identify the other parameters. The fixed and random effects and the distributional assumptions were as described above for the binary threshold model.

Poisson Model. A mixed effects Poisson model was also fitted to NMAST. Poisson models, which are appropriate for count data in the case of rare events, impose more structure because the mean and variance of the conditional distribution of the observations, given the random effects, are assumed to be equal. In the Poisson model, the effects of differences in exposure time (i.e., DIM) were explored in preliminary analyses. Because this did not improve fit, these effects were ignored in subsequent modeling. The mixed Poisson model for NMAST assumed that the number of mastitis cases of a cow in calving year $i$, daughter of sire $j$ in herd $k$ followed a Poisson distribution, given the random effects, with parameter $\lambda_{i j k}$. Further,

$$
\log \left(\lambda_{i j k}\right)=\eta_{i j k}
$$

The distribution assumed for the vector of random effects (sires and herds) was as described previously, except that the corresponding variances were at the level of the log-Poisson parameters.

\section{Prediction of Random Effects}

The best predictor for minimum mean squared error was the conditional expectation (Bulmer, 1980). For generalized linear mixed models (e.g., probit and Poisson), predictions of the random effects corresponded to the mode of their conditional distribution, given the parameters evaluated at their maximum likelihood estimates (Doran et al., 2007). For the ordinal threshold model, the random effect predictions were obtained as the mean of Gibbs samples drawn from the joint posterior distribution.

\section{Heritability Estimates}

Heritability of BINMAST and NMAST was assessed for the probit and ordinal threshold models as 


$$
h^{2}=\frac{4 \sigma_{s}^{2}}{1+\sigma_{s}^{2}+\sigma_{h}^{2}} .
$$

In the ordinal threshold model, heritability was estimated as the mean of its posterior distribution. All variance components are expressed relative to the residual variance, which is set to 1 in the threshold models, as mentioned earlier. For nonlinear models, variance components do not show the same additive partition. For the Poisson model, Foulley et al. (1987) suggested a heritability approximation in the $\eta$-scale (log scale), which, in this case, would be

$$
h_{\eta}^{2}=\frac{4 \sigma_{s}^{2}}{\sigma_{s}^{2}+\sigma_{h}^{2}+\lambda^{-1}},
$$

where $\lambda$ is the average Poisson parameter for the entire risk period. In this study, $\lambda_{i j k}$ was a subject specific parameter and was evaluated at the median of the population.

\section{Software}

The probit and Poisson models were fitted using the pedigreemm package (Bates and Vazquez, 2008) created in the $\mathrm{R}$ environment ( $\mathrm{R}$ Development Core Team, 2008). This procedure uses an approximation to maximum likelihood for estimation of fixed effects and variance components by using covariances between sires that are attributable to additive genetic relationships. The incidence matrix $\left(\mathbf{Z}_{s}\right)$ of sire effects was multiplied by the appropriate component of a Cholesky decomposition of the additive genetic relationship matrix, leaving the transformed sire effects uncorrelated (Harville and Callanan, 1989).

The ordinal threshold model was analyzed using a Fortran95 program developed by Y. M. Chang (Heringstad et al., 2006). The software uses a Bayesian implementation via the Gibbs sampler. The method of Raftery and Lewis (1992) and visual inspection of trace plots were used to assess the number of iterations and burn-in length required. A single long chain of 50,000 iterations was run, and the initial 10,000 samples were discarded as burn in. The remaining 40,000 samples were kept for posterior inference. This software works for sire models by using sire and maternal grandsire relationships instead of relationships through sires and dams.

\section{Predictive Ability Model Comparison}

The typical model comparison tools, such as the likelihood ratio test, Akaike's information criterion, or Bayesian information criterion, do not apply to different generalizations of the linear predictor, which have different scales and response variables (e.g., BINMAST, NMAST). The probit, ordinal threshold, and Poisson models differ in their likelihood function and response variable. A comparison between a survival and linear model (where the response variables differed: time to first mastitis case and absence or presence of mastitis, respectively) was performed previously (Carlén et al., 2005). In this study, however, there is not a formal quantification of model performance. A 5-fold cross-validation was carried out to evaluate predictive ability of the models, with 2 different loss functions. One of the loss functions evaluated mean squared error of prediction (MSEP), whereas the other was a cost-based function. In the 5-fold cross-validation, data were repeatedly split into 2 random subsets, one with four-fifths of the total for training and the other with one-fifth for testing with every sire represented in each subset.

MSEP. The MSEP was estimated for each model as follows:

$\operatorname{MSEP}($ model $)=\frac{1}{n} \sum_{f \in F} \sum_{q=1}^{n_{f}}\left[\hat{E}\left(y_{q} \mid \mathbf{x}_{q}^{\prime} \boldsymbol{\beta}^{(-f)}+\mathbf{z}_{q}^{\prime} \mathbf{u}^{(-f)}\right)-y_{q}\right]^{2}$

Here, $y_{q}$ is the observed value of datum $q$, with both BINMAST and NMAST used in all incidences to make the comparison valid; $n_{f}$ is the number of observations being predicted in subset $f$. Note that $\sum_{f \in F} n_{f}=44,908$, the total number of observations. Further, $\hat{E}($.$) repre-$ sents the predicted value of $y_{q}$ from the appropriate model with fixed and random effects excluding the subset $f$ containing the $q$ th datum. The expectation was computed as given below, where $\boldsymbol{\beta}^{(-f)}$ and $\mathbf{u}^{(-f)}$ denote the fixed and random effects estimated from the data excluding the observation to be predicted, and $\mathbf{x}_{q}^{\prime}$ and $\mathbf{z}_{q}^{\prime}$ are incidence row vectors. The predicted expectations had the following form:

1) Probit model:

$$
E\left(y_{q}\right)=\boldsymbol{\Phi}\left(\mathbf{x}_{q}^{\prime} \boldsymbol{\beta}^{(-f)}+\mathbf{z}_{q}^{\prime} \mathbf{u}^{(-f)}\right)
$$

2) Ordinal threshold model:

$$
E\left(y_{q}\right)=\sum_{m=0}^{5} m\left[\begin{array}{l}
\boldsymbol{\Phi}\left(t_{k}-\mathbf{x}_{q}^{\prime} \boldsymbol{\beta}^{(-f)}-\mathbf{z}_{q}^{\prime} \mathbf{u}^{(-f)}\right) \\
-\boldsymbol{\Phi}\left(t_{k-1}-\mathbf{x}_{q}^{\prime} \boldsymbol{\beta}^{(-f)}-\mathbf{z}_{q}^{\prime} \mathbf{u}^{(-f)}\right)
\end{array}\right],
$$


Table 1. Estimated mastitis cost per lactation by the absence or presence of clinical mastitis (BINMAST) or by the number of episodes of mastitis (NMAST), including total cost, discarded milk cost, drug cost, extra labor cost, veterinary cost, and reduced milk production per lactation

\begin{tabular}{lcccccc}
\hline & & \multicolumn{5}{c}{ NMAST } \\
\cline { 3 - 7 } Item & BINMAST & 1 & 2 & 3 & 4 & 5 \\
\hline Total cost $(\$)$ & 316.7 & 197.6 & 516.1 & 628.7 & $1,330.8$ & $1,183.1$ \\
Discarded milk $(\$)$ & 77.1 & 77.1 & 77.1 & 77.1 & 77.1 & 77.1 \\
Drugs $(\$)$ & 15.4 & 10 & 20 & 30 & 40 & 50 \\
Labor $(\$)$ & 23.1 & 15 & 30 & 45 & 60 & 75 \\
Veterinary costs $(\$)$ & 3.1 & 2 & 4 & 6 & 8 & 10 \\
Reduced milk $(\$)$ & 203.0 & 93.5 & 385.0 & 470.6 & $1,145.7$ & 971.0 \\
Reduced milk $(\mathrm{kg})$ & 659 & 333 & 687 & 560 & 1,022 & 693 \\
\hline
\end{tabular}

where $m=0,1, \ldots, 5$ denote the 6 possible categories of the response variable; and

3) Poisson model:

$$
E\left(y_{q}\right)=\exp \left(\mathbf{x}_{q}^{\prime} \boldsymbol{\beta}^{(-f)}+\mathbf{z}_{q}^{\prime} \mathbf{u}^{(-f)}\right)=\lambda_{i j k} .
$$

The mean squared error of a model is the sum of the variance and the squared bias. A model with smaller MSEP has better predictive abilities and should be preferred.

Cost-Based Loss Function. A cost-based loss function, defined as the mean of the absolute difference between observed mastitis cost and predicted mastitis cost, was used to evaluate each model. The observed mastitis costs were calculated using the costs reported in Guard (2008) and the observed number of cases for each cow. Costs of drugs, veterinary fees, and extra labor (Table 1) depended on the observed number of mastitis cases per lactation, whereas costs of discarded milk represented an average loss per lactation. A similar approximation was used to assess model performance, using the true cost of treatments for each cow (PérezCabal et al., 2009). Reduced milk production per lactation, according to the number of mastitis episodes, was estimated with a linear mixed model, using information from lactations with at least 215 DIM as follows:

$$
m_{i l k} k_{i j k l}=\alpha+C Y_{i}+S_{j}+H_{k}+N M A S T_{i j k l}+\varepsilon_{i j k l},
$$

where milk $_{i j k l}$ is the 305 -d standardized milk production of a daughter $l$ of the $j$ th sire, calving year $i$, in herd $k$; $\alpha$ is an effect common to all lactations; and $C Y, S, H$, $N M A S T$, and $\varepsilon_{i j k l}$ are as defined previously. Milk price was $\$ 0.280 / \mathrm{kg}$ after hauling charges (VanRaden and Multi-State Project S-1008, 2006). Observed mastitis costs, by number of episodes of mastitis and per infected lactation, were across individual cows.

Predicted mastitis costs were estimated with the predicted expectation of the cross-validation, $E\left(y_{i j k l}\right)$, times the cost of a cow either becoming infected with mastitis or having $m$ mastitis cases for the models with binary and count response variables, respectively. The loss function associated with record $y_{i j k l}$ was as follows:

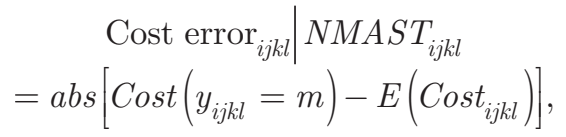

where $\operatorname{Cost}\left(y_{i j k l}=m\right)$ is the average observed cost per lactation with $m$ cases of mastitis (Table 1 ); and

$$
E\left(\operatorname{Cost}_{i j k l}\right)=\sum_{m \in M} P\left(y_{i j k l}=m\right) \times \operatorname{Cost}\left(y_{i j k l}=m\right),
$$

where $E\left(\right.$ Cost $\left._{i j k l}\right)$ is the expected cost of mastitis for the model prediction, and $P\left(y_{i j k l}=m\right)$ is the probability of $m$ mastitis cases. For the probit model, $y_{i j k l}$ is BINMAS$T_{i j k l}(m=0,1)$, whereas for the ordinal threshold and Poisson models, $y_{i j k l}$ is $N M A S T_{i j k l}(m=0, \ldots, 5)$.

\section{RESULTS AND DISCUSSION}

\section{Estimates of Variances and Heritability}

The variance components estimated with each model vary in scale (Table 2 ). For the Poisson model, variances are in the $\log (\lambda)$ scale and for the threshold models, variances are in the latent variable scale for either BINMAST or NMAST. Additionally, the Poisson model does not have a residual, whereas in the threshold models (probit for BINMAST and ordinal for NMAST), the residual variance is set to 1 . Therefore, it is not possible to compare variance components between models. Environmental factors have a major effect on mastitis incidence. Effects related to the herd grouping factor (e.g., hygiene, management practices, type and number of pathogens) capture most of the variation in all models. The heritability estimate from the probit model was 0.061 , which agrees with previous 

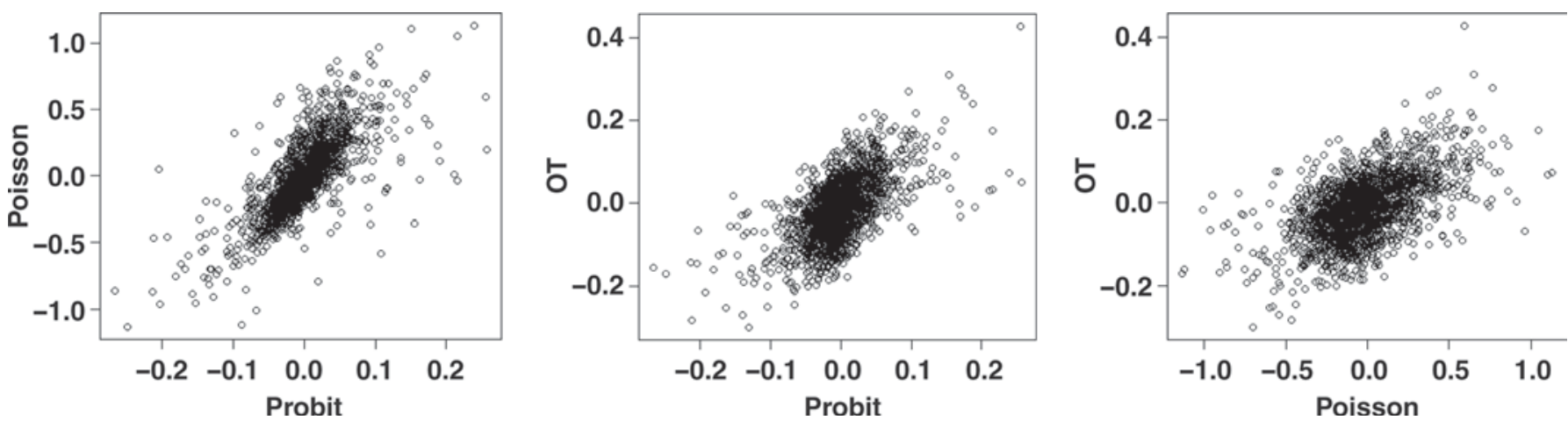

Figure 1. Sire effects association between the Poisson and probit model (left panel), the probit and ordinal threshold model (OT; center panel), and the Poisson and OT model (right panel).

reports varying between 0.06 and 0.12 (Heringstad et al., 2000). For the ordinal threshold model in this study, the estimated heritability was 0.085 , which is very close to previous results with Norwegian Red cattle (Heringstad et al., 2006). The $\lambda$ parameter in the Poisson model is year $\times$ herd $\times$ sire specific and its estimate ranged between 0 and 3.66. The Poisson heritability, evaluated at the median $\lambda(0.158)$, yielded a heritability estimate of 0.132 . A previously published estimate of heritability in a Poisson model in Norwegian Red cattle was 0.07 (Vazquez et al., 2009). Because $\lambda$ is a year $\times$ herd $\times$ sire-specific parameter, rather than a population parameter, this estimate of heritability is questionable. In general, the concept of heritability in nonadditive partitions of the variance is problematic, as described in Vazquez (2007).

\section{Prediction of Random Effects}

Predictions of sire effects for BINMAST when using the probit model ranged from -0.266 to 0.257 in the scale of the latent variable. Predictions of sire effects for NMAST with the ordinal threshold model ranged from -0.299 to 0.427 , also in the scale of the latent variable; for the Poisson model, these estimates ranged from -1.133 to 1.130 in the $\log (\lambda)$ scale. Correlations between estimated sire effects are shown in Table 3. The correlation between estimates from the probit and Poisson models was the largest, followed by those of the ordinal threshold and probit models. Associations between estimated sire effects are plotted in Figure 1. Estimated herd effects were highly correlated between the 3 models (0.98 to 0.99 ), probably because of the large number of records per herd (mean 279.5). The range for predicted herd effects in the probit model was between -1.096 and 1.319 in the liability scale; in the ordinal threshold model, the range was between -1.204 and 1.348 in the liability scale; and in the Poisson model, the range was between -2.168 and 2.284 in the $\log (\lambda)$ scale.

Finally, associations of the fitted values for the 3 models are shown in Figure 2. Fitted values were defined as the expected values of the data $y_{i j k l}$ given the estimated fixed effects and predicted random effects. The correlation between the fitted values for each model is also shown in Table 3 . The 2 threshold models (probit and ordinal threshold) were very similar with respect to fitted values $(r=0.97)$. This result is not surprising, because both models depend on a single linear predictor that was defined equivalently, but they vary in the number of thresholds to be estimated. Expected values from the various models differed, especially in the range of values predicted by the models. The Poisson model gave a larger range of predicted values, ranging from 0 to 3.66, in agreement with the results of Vazquez et al. (2009). The ordinal threshold model gave a range of predicted values from 0 to 1.40 when predicting NMAST, and the probit model gave

Table 2. Estimates of variance components for mastitis measured as a binary character and number of episodes of mastitis per lactation in the 3 models

\begin{tabular}{lccc}
\hline Variance component & Probit (BINMAST) & Ordinal threshold (NMAST) & Poisson (NMAST) \\
\hline Sire & 0.0193 & 0.0282 & 0.2510 \\
Herd & 0.2534 & 0.2975 & 0.9696 \\
Residual & 1 & 1 & - \\
Variance ratio of herd:sire & 13.1 & 10.5 & 3.9 \\
Heritability & 0.061 & 0.085 & 0.132 \\
\hline
\end{tabular}


Table 3. Correlation between predictions of sire effects (below the diagonal), and correlation between fitted values (above the diagonal) for the 3 models

\begin{tabular}{lccc}
\hline Item & Probit & Ordinal threshold & Poisson \\
\hline Probit & - & 0.97 & 0.91 \\
Ordinal threshold & 0.61 & - & 0.91 \\
Poisson & 0.74 & 0.55 & - \\
\hline
\end{tabular}

a range of predicted values from 0 to 0.83 when predicting BINMAST. These results show how similar the ordinal threshold and probit models are. The ordinal threshold model led to an extreme category problem for some levels of the fixed effects because of the large number of zeros.

\section{Predictive Ability in Cross-Validation}

MSEP. The MSEP results for BINMAST and NMAST are shown in Table 4. The overall MSEP is not very meaningful because it averages MSEP for different situations. This was also observed in a Norwegian population of cows (Vazquez et al., 2009), where the overall MSEP was very similar between the Bernoulli, Poison, and linear models for count or binary responses (0.351, 0.346, 0.345, and 0.350 , respectively), but results within each subcategory (e.g., sick and healthy cows) presented important differences. As in previous studies, the model for binary variables provided better fit when evaluating healthy cows compared with the models for event counts (Vazquez, 2007; Vazquez et al., 2009). The model for binary variables fits only 2 categories and can accommodate zeros better than any other model. Observations corresponding to sick cows and to particular categories of mastitis counts were better predicted with the models that analyzed case counts than with the probit model. The Poisson model generally provides the best fit for all categories of the count
Table 4. Mean square error of prediction for the 5 -fold cross-validation study $^{1}$

\begin{tabular}{lccc}
\hline Item & Probit & Ordinal threshold & Poisson \\
\hline A. BINMAST & & & \\
All & 0.12 & 0.13 & 0.15 \\
Healthy & 0.03 & 0.07 & 0.09 \\
Sick & 0.60 & 0.47 & 0.46 \\
B. NMAST & & & \\
All & 0.50 & 0.48 & 0.48 \\
Healthy & 0.03 & 0.07 & 0.09 \\
Sick & 3.03 & 2.68 & 2.58 \\
1 case of mastitis & 0.62 & 0.50 & 0.50 \\
2 cases of mastitis & 3.08 & 2.64 & 2.53 \\
3 cases of mastitis & 7.39 & 6.52 & 6.15 \\
4 cases of mastitis & 13.82 & 12.59 & 11.93 \\
5 cases of mastitis & 21.90 & 20.01 & 19.16 \\
6 cases of mastitis & 31.91 & 29.16 & 27.97 \\
\hline
\end{tabular}

${ }^{1} \mathrm{~A}=$ presence or absence of mastitis as a response (BINMAST); $\mathrm{B}=$ number of cases of mastitis as a response (NMAST).

variable but provides a poorer fit for healthy cows. The results for the binary and Poisson models agree with those obtained by Vazquez et al. (2009). Overall, the probit model is more accurate for detecting sires with healthy daughters, but the Poisson model is preferred for detecting sires with daughters with multiple cases of mastitis, followed by the ordinal threshold model.

Cost-Based Loss Function. The estimated reduction in milk tends to increase with the number of mastitis events (Table 1). Income reduction caused by reduced milk production for NMAST and BINMAST has the same tendency. The total mastitis cost was estimated by combining this result with discarded milk, drugs, labor, and veterinary costs, considering the number of mastitis events. The cost-based loss function (Table 5) showed that the ordinal threshold model had better predictive ability than the probit model, even for healthy cows. The cost loss function changed the predictive ability of the probit model in healthy cows with respect to the result from the MSEP loss function,
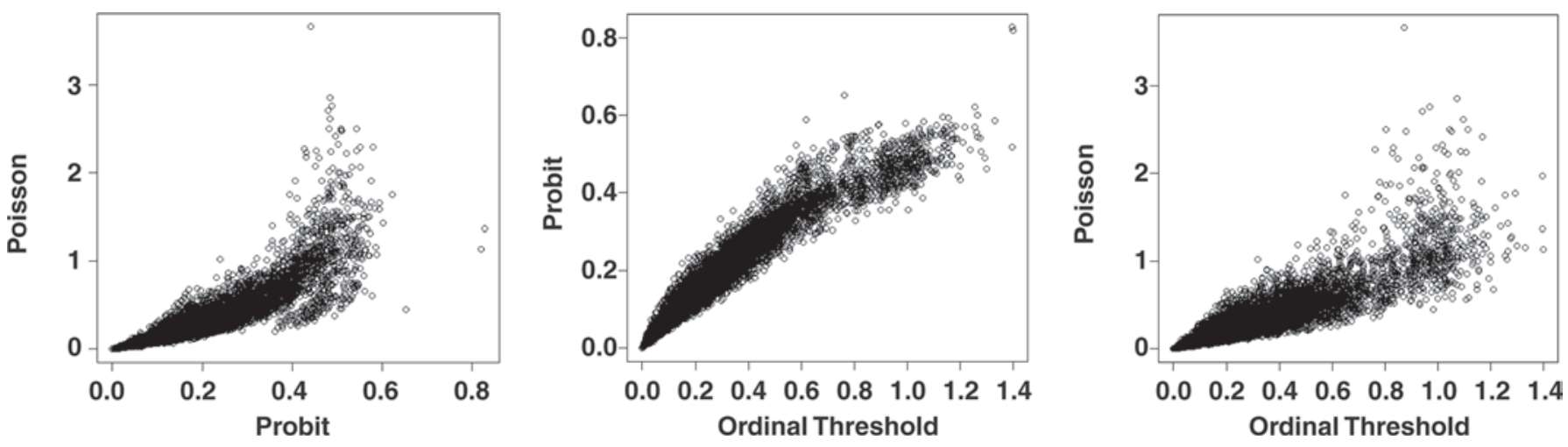

Figure 2. Association between expected values for the Poisson and probit model (left panel), the probit and ordinal threshold model (center panel), and the Poisson and ordinal threshold model (right panel). 
Table 5. Cost loss function (in $\$ /$ lactation) for prediction of mastitis costs using the presence or absence of clinical mastitis (BINMAST) or number of episodes of mastitis per lactation (NMAST) as the response variable

\begin{tabular}{lccc}
\hline Item & $\begin{array}{c}\text { Probit } \\
(\text { BINMAST) }\end{array}$ & $\begin{array}{c}\text { Ordinal threshold } \\
\text { (NMAST) }\end{array}$ & $\begin{array}{c}\text { Poisson } \\
\text { (NMAST) }\end{array}$ \\
\hline All & 76.2 & 74.4 & 75.5 \\
Healthy & 44.7 & 42.7 & 45.0 \\
Infected & 246.6 & 245.8 & 240.5 \\
NMAST $=1$ & - & 128.1 & 127.7 \\
NMAST $=2$ & - & 434.0 & 417.4 \\
NMAST $=3$ & - & 532.7 & 504.7 \\
NMAST $=4$ & - & $1,234.6$ & $1,203.1$ \\
NMAST $=5$ & - & $1,072.2$ & $1,039.0$ \\
\hline
\end{tabular}

because the average cost attributable to mastitis considered the number of cases per lactation. The probit model still provided better predictive ability than the Poisson model for healthy cows, but not for infected cows. Another study in a Spanish population of cows reported that a cost-based loss function favored a Poisson model relative to a Bernoulli model for mastitis (Pérez-Cabal et al., 2009). In agreement with the above discussion, the Poisson model had better performance than the ordinal threshold model when predicting infected cows and a count of mastitis cases, but was poorer for prediction of healthy cows.

\section{CONCLUSIONS}

The cross-validation study evaluated with 2 different loss functions suggested that threshold models were suitable for identifying sires that were likely to have healthy daughters. The binomial model yielded more accurate predictions, but the cost-based estimation favored the ordinal threshold model. On the other hand, results of the present study suggested that the Poisson model and, to a lesser extent, the ordinal threshold model were more suitable for identifying sires that were likely to have infected daughters, particularly those sires whose daughters were highly susceptible to mastitis and were likely to have multiple clinical episodes per lactation.

\section{ACKNOWLEDGMENTS}

The list of comments used to identify mastitis cases was developed in collaboration with Nigel Cook from the University of Wisconsin-Madison School of Veterinary Medicine. Data were generously provided by Alta Genetics (Watertown, WI). Partial financial support of K. Weigel by the National Association of Animal Breeders (Columbia, MO) is gratefully acknowledged. The lead author, A. Vazquez, was supported by USDAARS research contract 58-1265-3-151. Support by the
Wisconsin Agriculture Experiment Station (Madison) and by grant DMS-NSF DMS-044371 to D. Gianola are acknowledged.

\section{REFERENCES}

Bates, D. M., and A. I. Vazquez. 2008. Pedigree-based mixed models project. http://pedigreemm.r-forge.r-project.org/ Accessed Jan. 2009

Bulmer, M. G. 1980. The Mathematical Theory of Quantitative Genetics. Oxford, New York, NY.

Carlén, E., M. del P. Schneider, and E. Strandberg. 2005. Comparison between linear models and survival analysis for genetic evaluation of clinical mastitis in dairy cattle. J. Dairy Sci. 88:797-803.

Doran, H., D. M. Bates, P. Bliese, and M. Dowling. 2007. Estimating the multilevel Rasch model: With the lme4 package. J. Stat. Softw. 20:1-18.

Foulley, J. L., D. Gianola, and S. Im. 1987. Genetic evaluation for traits distributed as Poisson binomial with reference to reproductive characters. Theor. Appl. Genet. 73:870-877.

Gasqui, P., and J. Barnouin. 2003. Statistical modeling for clinical mastitis in the dairy cow: Problems and solutions. Vet. Res. 34:493-505.

Gianola, D. 1982. Theory and analysis of threshold characters. J. Anim. Sci. 54:1079-1099.

Gianola, D., and J. L. Foulley. 1983. Sire evaluation for ordered categorical data with a threshold model. Genet. Sel. Evol. $15: 201-224$.

Guard, C. 2008. The costs of common diseases of dairy cattle. http:// veterinarycalendar.dvm360.com/avhc/Veterinary+Food+Animal/ The-costs-of-common-diseases-of-dairy-cattle-Proce/ ArticleStandard/Article/detail/586496 Accessed July 2009.

Harville, D. A., and T. P. Callanan. 1989. Computational aspects of likelihood-based inference for variance components. Pages 136-176 in Advances in Statistical Methods for Genetic Improvement of Livestock. D. Gianola and K. Hammond, ed. Springer-Verlag, Berlin, Germany.

Heringstad, B., Y. M. Chang, I. M. Andersen-Ranberg, and D. Gianola. 2006. Genetic analysis of number of mastitis cases and number of services to conception using a censored threshold model. J. Dairy Sci. 89:4042-4048.

Heringstad, B., Y. M. Chang, D. Gianola, and G. Klemetsdal. 2004. Multivariate threshold model analysis of clinical mastitis in multiparous Norwegian dairy cattle. J. Dairy Sci. 87:3038-3046.

Heringstad, B., G. Klemetsdal, and J. Ruane. 2000. Selection for mastitis resistance in dairy cattle $-\mathrm{A}$ review with focus on the situation in the Nordic countries. Livest. Prod. Sci. 64:95-106.

Pérez-Cabal, M. A., G. de los Campos, A. I. Vazquez, D. Gianola, G. J. M. Rosa, K. A. Weigel, and R. Alenda. 2009. Genetic evaluation of susceptibility to clinical mastitis in Spanish Holstein cows. J. Dairy Sci. 92:3472-3480.

R Development Core Team. 2008. R: A language and environment for statistical computing. R Foundation for Statistical Computing, Vienna, Austria. http://www.R-project.org Accessed Sep. 2009.

Raftery, A. E., and S. M. Lewis. 1992. [Practical Markov chain Monte Carlo]: Comment: One long run with diagnostics: Implementation strategies for Markov chain Monte Carlo. Stat. Sci. 7:493-497.

Rekaya, R., S. L. Rodriguez-Zas, D. Gianola, and G. E. Shook. 1998. Test-day models for longitudinal binary responses: An application to mastitis in Holsteins. Paper presented at the 49th Annu. Meet. Eur. Assoc. Anim. Prod., Warsaw, Poland.

Saebo, S., T. Almoy, A. H. Aastveit, B. Heringstad, and G. Klemetsdal. 2002. Modelling time to first treatment of clinical mastitis as first passage times of stochastic processes. In Proc. 7th World Congr. Genet. Appl. Livest. Prod., Montpellier, France [CD-ROM Commun. No. 16-16]. INRA, Castanet-Tolosan, France.

Shook, G. E. 1982. Approaches to summarizing somatic cell count which improve interpretability. Proc. Natl. Mastitis Counc. 21:150-166.

VanRaden, P. M., and Multi-State Project S-1008. 2006. Net merit as a measure of lifetime profit: 2006 revision. http://aipl.arsusda. 
gov/reference/nmcalc.htm\#Updated\%20economic\%20values Accessed Dec. 2008.

Vazquez, A. I. 2007. Analysis of number of episodes of clinical mastitis in Norwegian Red and Holstein cows with Poisson and categorical data mixed models. MS Thesis. University of Wisconsin, Madison.

Vazquez, A. I., D. Gianola, D. M. Bates, K. A. Weigel, and B. Heringstad. 2009. Assessment of Poisson, logit, and linear models for genetic analysis of clinical mastitis in Norwegian Red cows. J. Dairy Sci. 92:739-748.
Zwald, N. R. 2004. Development and categorization of systems for evaluating health, fertility, and disease resistance in dairy sires. $\mathrm{PhD}$ Thesis. University of Wisconsin, Madison.

Zwald, N. R., K. A. Weigel, Y. M. Chang, R. D. Welper, and J. S. Clay. 2004. Genetic selection for health traits using producerrecorded data. I. Incidence rates, heritability estimates, and sire breeding values. J. Dairy Sci. 87:4287-4294. 